Zbigniew Jelonek

\title{
On the preimage of a sphere by a polynomial mapping
}

Received: 4 November 2019 / Accepted: 11 August 2020 /

Published online: 17 August 2020

Abstract. Let $X$ be an irreducible complex affine variety of dimension greater than one and let $f: X \rightarrow \mathbb{C}^{m}$ be a polynomial mapping. Let $|*|$ be a semialgebraic norm on $\mathbb{C}^{m}$. Then for $R$ large enough the sets $f^{-1}\left(B_{R}\right), f^{-1}\left(S_{R}\right), X \backslash f^{-1}\left(B_{R}\right)$ are all connected, where $B_{R}=\left\{z \in \mathbb{C}^{m}:|z| \leq R\right\}$ and $S_{R}=\left\{z \in \mathbb{C}^{m}:|z|=R\right\}$. As an application we show that if $F$ is a counterexample to the Jacobian Conjecture, then the non-properness set of $F$ has a non-trivial link at infinity.

\section{Introduction}

Let $\mathbb{K}=\mathbb{R}$ or $\mathbb{K}=\mathbb{C}$. Despite of the fact that polynomial mappings are one of the most basic tools in real and complex analysis, their topological properties are not well understood. Let $|*|$ be a semi-algebraic norm in $\mathbb{K}^{m}$ (e.g., $|x|=\sqrt{\sum_{i=1}^{m}\left|x_{i}\right|^{2}}$ or $\left.|x|=\sup _{i=1, \ldots, m}\left\{\left|x_{i}\right|\right\}\right)$. Let $S_{R}$ be the sphere of radius $R$ and $B_{R}$ the ball of radius $R$ in $\mathbb{K}^{m}$, i.e., $B_{R}=\left\{z \in \mathbb{K}^{m}:|z| \leq R\right\}$ and $S_{R}=\left\{z \in \mathbb{K}^{m}:|z|=R\right\}$. It is well known (see e.g. [7,9]) that if a polynomial mapping $f: \mathbb{K}^{n} \rightarrow \mathbb{K}^{m}$ $(n>1)$ is proper, then the set $f^{-1}\left(S_{R}\right)$ for large $R$ is connected. This is not true for an arbitrary real polynomial mapping $f: \mathbb{R}^{n} \rightarrow \mathbb{R}^{m}$. In particular it is easy to see that for $f: \mathbb{R}^{n} \rightarrow \mathbb{R}^{n}, f=\left(x_{1}^{2} x_{2} \ldots x_{n}, x_{1} x_{2}^{2} \ldots x_{n}, \ldots, x_{1} x_{2} \ldots x_{n}^{2}\right)$ the set $f^{-1}\left(S_{R}\right)$ has at least $2^{n}$ connected components. Indeed, $f^{-1}\left(S_{R}\right) \subset \mathbb{R}^{n} \backslash f^{-1}(0)=$ $\mathbb{R}^{n} \backslash\left\{x_{1} x_{2} \ldots x_{n}=0\right\}$. The set $\mathbb{R}^{n} \backslash\left\{x_{1} x_{2} \ldots x_{n}=0\right\}$ has $2^{n}$ connected components. Now in each of these components there is a point $\left(\epsilon_{1} r, \ldots, \epsilon_{n} r\right)$, where $r^{n+1}=R$ and $\epsilon_{k}= \pm 1$, which belongs to $f^{-1}\left(S_{R}\right)$. We have assumed here that on $\mathbb{R}^{n}$ we have the sup norm, but of course we can easily modify the proof for other norms.

We show however that the complex case is different and for every polynomial mapping $f: \mathbb{C}^{n} \rightarrow \mathbb{C}^{m}$ the preimage of a sufficiently large sphere is connected. In fact we prove more:

Author is partially supported by the grant of Narodowe Centrum Nauki Number 2019/33/B/ST1/00755

Z. Jelonek $(\bowtie)$ : Instytut Matematyczny, Polska Akademia Nauk, Śniadeckich 8, 00-656 Warsaw, Poland

Email: najelone@cyf-kr.edu.pl

Mathematics Subject Classification: 14R99 $\cdot$ 14P10 - 30C10

https://doi.org/10.1007/s00229-020-01239-6 
Theorem 1.1. Let $X$ be an irreducible complex affine variety of dimension greater than one and let $f: X \rightarrow \mathbb{C}^{m}$ be a polynomial mapping. Let $|*|$ be a semi-algebraic norm on $\mathbb{C}^{m}$. Then for $R$ large enough the sets $f^{-1}\left(B_{R}\right), f^{-1}\left(S_{R}\right), X \backslash f^{-1}\left(B_{R}\right)$ are all connected.

In particular we have:

Corollary 1.2. Let let $f: \mathbb{C}^{n} \rightarrow \mathbb{C}^{m}$ be a polynomial mapping. Let $|*|$ be a semialgebraic norm on $\mathbb{C}^{m}$. Then for $R$ large enough the sets $f^{-1}\left(B_{R}\right), f^{-1}\left(S_{R}\right)$, $\mathbb{C}^{n} \backslash f^{-1}\left(B_{R}\right)$ are all connected.

Proof. Note that for $n=1$ the mapping $f$ is either finite or constant and we can use [7,9]. Alternatively, we can note that for $X=\mathbb{C}$ Theorem 2.1 is still true.

As mentioned before, the last corollary is not true for an arbitrary real polynomial mapping $f: \mathbb{R}^{n} \rightarrow \mathbb{R}^{m}$. However, we believe that the following conjecture is true:

Conjecture. Let $f=\left(f_{1}, \ldots, f_{n}\right): \mathbb{R}^{n} \rightarrow \mathbb{R}^{n}$ be a polynomial dominant mapping (i.e., $\overline{f\left(\mathbb{R}^{n}\right)}=\mathbb{R}^{n}$ ) and $B(f)$ be the bifurcation set of $f$. Assume that:

(1) $\operatorname{codim} B(f) \geq 2$,

(2) $\operatorname{codim} f^{-1}(B(f)) \geq 2$.

Then for $R$ large enough the sets $f^{-1}\left(B_{R}\right), f^{-1}\left(S_{R}\right), \mathbb{R}^{n} \backslash f^{-1}\left(B_{R}\right)$ are all connected.

Let us recall that $B(f)=f(C(f)) \cup S_{f}$, where $C(f)$ denotes the set of critical points of $f$ and $S_{f}$ denotes the set of points at which $f$ is not proper (for the definition of $S_{f}$ see e.g., $\left.[3,4]\right)$.

Remark 1.3. It is not difficult to prove, that for every real polynomial mapping $f: \mathbb{R}^{n} \rightarrow \mathbb{R}^{m}$, the set $f^{-1}\left(B_{R}\right)$ is connected for $R$ large enough. Hence the difficult part of our Conjecture is to prove that the set $f^{-1}\left(S_{R}\right)$ is connected.

\section{Proof of Theorem 1.1}

First assume that $X$ is a normal irreducible variety. Let $X^{\prime}$ be a projective closure of $X$ and consider graph $(f) \subset X^{\prime} \times Y$, where $Y=\overline{f(X)}$. Denote by $\Gamma$ the normalization of the closure of $\operatorname{graph}(f)$ in $X^{\prime} \times Y$. We have the canonical proper projection $\pi: \Gamma \rightarrow Y$. We can assume that $X \subset \Gamma$ and $f=\left.\pi\right|_{X}$, hence we can treat the mapping $\pi$ as an extension of $f$ to $\Gamma$. In further we will identify $\pi$ with $f$. We will need the following important theorem (for a proof see [2, pp. 141-142]):

Stein Factorization Theorem. Let $f: \Gamma \rightarrow Y$ be a proper dominating morphism of irreducible algebraic varieties. If $\Gamma$ is normal, then there exists a normal variety $W$, a finite morphism $\mu: W \rightarrow Y$, and a surjective morphism $g: \Gamma \rightarrow W$ with connected fibers such that $f=\mu \circ g$. 
Note that in our case the variety $Y$ is affine, so $W$ is affine, too. Consider the mapping $F=\iota \circ \mu: W \rightarrow Y \subset \mathbb{C}^{n}$, where $\iota: Y \rightarrow \mathbb{C}^{n}$ is the inclusion. Let $|*|$ be a semi-algebraic norm on $\mathbb{C}^{n}$. We show that for $R$ large enough the sets $F^{-1}\left(B_{R}\right), F^{-1}\left(S_{R}\right), W \backslash F^{-1}\left(B_{R}\right)$ are all connected. Put $V(R):=F^{-1}\left(B_{R}\right)$. Let us examine the behavior of the sets $V(R)$. To do this we introduce the function $\psi: W \ni y \mapsto|F(y)| \in \mathbb{R}_{+}$(here $\mathbb{R}_{+}=\{t \in \mathbb{R}: t \geq 0\}$ ). Note that the set $W$ is connected and semialgebraic. We have $V(R)=\psi^{-1}([0, R])$. Since $\psi$ is semialgebraic, it has only a finite number of bifurcation values $P_{0}=0<P_{1}<$ $\cdots<P_{r} \in \mathbb{R}_{+}$and outside them $\psi$ is a locally trivial fibration by the Thom Isotopy Lemma (see [1, Theorem 2.7.1]). For technical reasons we put additionally $P_{r+1}=+\infty$.

Note that the number of connected components of $V(R)$ can change only if $\psi$ passes through a bifurcation value. Indeed, if $R_{2}>R_{1}$ and $\left[R_{1}, R_{2}\right] \subset\left(P_{i}, P_{i+1}\right)$, then $V\left(R_{2}\right) \backslash V\left(R_{1}\right) \cong\left(R_{1}, R_{2}\right] \times F^{i}$, where $F^{i}$ is a generic fiber of $\psi$ over $I_{i}=$ $\left(P_{i}, P_{i+1}\right)$. In particular $V\left(R_{1}\right)$ is a deformation retract of $V\left(R_{2}\right)$ and they have the same number of connected components. Hence after we pass through the last critical value, the number of connected component of $V(R)$ remains constant.

Fix $R_{0}>P_{r}$. The set $W \backslash V\left(R_{0}\right)$ is homeomorphic to the product $\left(R_{0}, \infty\right) \times F_{r}$. In particular $V\left(R_{0}\right)$ is a deformation retract of $W$. Hence $V\left(R_{0}\right)$ has only one component.

Now we examine the behavior of $W \backslash V(R)$. We need the following result which follows from [8, Theorem 6.3 ]:

Theorem 2.1. Let $X$ be a connected normal Stein variety of dimension greater than one and let $K \subset X$ be a compact set. Then the set $X \backslash K$ has only one unbounded component.

Take $R>R_{0}$. Let us note that $V(R)$ is a compact set and $W \backslash V(R) \cong(R, \infty) \times$ $F_{r}$. Hence all connected components of $W \backslash V(R)$ are unbounded. This means by Theorem 2.1 that there is only one such component, i.e., $W \backslash F^{-1}\left(B_{R}\right)$ is connected. But $W \backslash F^{-1}\left(B_{R}\right) \cong(R,+\infty) \times F^{-1}\left(S_{R}\right)$, so $F^{-1}\left(S_{R}\right)$ is connected, too.

Because the mapping $g$ is surjective and has connected fibers we conclude that the sets $\pi^{-1}\left(B_{R}\right), \pi^{-1}\left(S_{R}\right), \Gamma \backslash \pi^{-1}\left(B_{R}\right)$ are connected, too. The following statement is true (see $[6$, chapter VI]):

Theorem 2.2. Let $X$ be a normal analytic space and $G \subset X$ be an open connected set. Assume that $Q \subset G$ is a nowhere-dense analytic subset of $G$. Then $G \backslash Q$ is connected.

Denote $Q=\Gamma \backslash X$. Since the set $\Gamma \backslash \pi^{-1}\left(B_{R}\right)$ is connected and the space $\Gamma$ is normal, the set $\left(\Gamma \backslash \pi^{-1}\left(B_{R}\right)\right) \backslash Q$ is also connected (see Theorem 2.2). This means that $X \backslash f^{-1}\left(B_{R}\right)$ is connected.

We can repeat our previous argument for the function $\phi: X \ni x \mapsto|f(x)| \in$ $\mathbb{R}_{+}$to prove that the sets $f^{-1}\left(S_{R}\right)$ and $f^{-1}\left(B_{R}\right)$ are connected, for $R$ large enough.

Finally, let $X$ be an arbitrary irreducible affine variety and $f: X \rightarrow \mathbb{C}^{m}$ be a polynomial mapping. Let $q: X^{\prime} \rightarrow X$ be the normalization and take $F=f \circ q$. Note that $X^{\prime}$ is also an affine irreducible variety. By our previous result the sets 
$F^{-1}\left(B_{R}\right), F^{-1}\left(S_{R}\right), X^{\prime} \backslash F^{-1}\left(B_{R}\right)$ are connected for $R$ large enough. Since $q$ is surjective, the sets $f^{-1}\left(B_{R}\right), f^{-1}\left(S_{R}\right), X \backslash f^{-1}\left(B_{R}\right)$ are also connected.

\section{Application}

In this section we apply our result to the Jacobian Conjecture. Let us recall famous:

Jacobian Conjecture. Let $F: \mathbb{C}^{n} \rightarrow \mathbb{C}^{n}$ be a polynomial mapping with a nowhere vanishing jacobian. Then $F$ is a biholomorphism.

If $F$ is a counterexample to the Jacobian Conjecture, then $F$ is non-proper and its non-properness set $S_{F}$ is non-empty and hence it is a hypersurface (see e.g., [3-5]). Let us recall that $S_{F}=\left\{y \in \mathbb{C}^{n}\right.$ : there is a sequence $x_{n} \rightarrow \infty$ such that $F\left(x_{n}\right) \rightarrow y$ \} (see e.g. [3,4]). We show here that the set $S_{F}$ has a non-trivial link at infinity.

Definition 3.1. Let $X \subset \mathbb{C}^{n}$ be an algebraic hypersurface. For $R$ large enough the topology of the pair $\left(S_{R}, S_{R} \cap X\right)$ does not depend on $R$ (see section 2). We call the pair $\left(S_{R}, S_{R} \cap X\right)$ the link at infinity of $X$. We say that this link is trivial if $\pi_{1}\left(S_{R} \backslash S_{R} \cap X\right)=\mathbb{Z}$.

From Theorem 1.1 it follows that the link of irreducible variety is connected if $n \geq 3$. It is easy to see, that it is also connected if $n=2$ and $X$ is a parametric curve. Of course every trivial link has to be connected. We have:

Lemma 3.2. Assume that the affine hypersurface $X \subset \mathbb{C}^{n}$ has trivial link at infinity. Then for sufficiently large $R$, for a smooth point $a \in S_{R} \cap X$ and a neighborhood $U_{a} \subset S_{R}$ there is a loop $\gamma \subset U_{a}$ which generates $H_{1}\left(S_{R} \backslash X\right)$.

Proof. Since $\pi_{1}\left(S_{R} \backslash S_{R} \cap X\right)=\mathbb{Z}$ we have $H_{1}\left(S_{R} \backslash S_{R} \cap X\right)=\mathbb{Z}$. We show that the latter group is generated by a small loop around $S_{R} \cap X$.

Indeed, consider the function $\psi: \mathbb{C}^{n} \ni y \mapsto|y| \in \mathbb{R}_{+}$. The pair $\left(\mathbb{C}^{n}, X\right)$ has a finite Whitney algebraic stratification $\mathcal{S}=\left\{\mathbb{C}^{n} \backslash X, S_{1}, \ldots, S_{k}\right\}$, where $X=$ $\bigcup_{i=1}^{r} S_{i}$. Since $\psi$ is semialgebraic, it has only a finite number of bifurcation values $P_{0}=0<P_{1}<\cdots<P_{r} \in \mathbb{R}_{+}$on $\mathcal{S}$ and outside them $\psi$ is a locally trivial fibration which preserves strata (see [1, Theorem 2.7.1]). In particular the pair $\left(S_{R}, S_{R} \cap X\right)$ is a deformation retract of $\left(\mathbb{C}^{n} \backslash \operatorname{Int}\left(B_{R}\right), X \backslash \operatorname{Int}\left(B_{R}\right)\right)$ for $R$ large enough. Let us denote this retraction by $r$.

Since $X$ is an irreducible hypersurface (it has a trivial link!), we have $H_{1}\left(\mathbb{C}^{n} \backslash X\right)=\mathbb{Z}$. Let $\alpha$ be a small loop around $a$ which is a generator of $H_{1}\left(\mathbb{C}^{n} \backslash X\right)$ (for details see [10], Lemma 2.3, a). We can choose $\alpha$ so small, that $r(\alpha):=\gamma \subset U_{a}$. Since $r$ induces the isomorphism

$$
r_{*}: H_{1}\left(\left(\mathbb{C}^{n} \backslash X\right) \backslash \operatorname{Int}\left(B_{R}\right)\right) \rightarrow H_{1}\left(S_{R} \backslash S_{R} \cap X\right)
$$

we see that $\alpha$ is homologous to $\gamma$ in $\mathbb{C}^{n} \backslash X$. Now consider the mapping

$$
\mathbb{Z}=H_{1}\left(S_{R} \backslash S_{R} \cap X\right) \rightarrow H_{1}\left(\mathbb{C}^{n} \backslash X\right)=\mathbb{Z} .
$$

Since $\gamma$ goes to the generator of $H_{1}\left(\mathbb{C}^{n} \backslash X\right)$, it has to be generator of $H_{1}\left(S_{R} \backslash S_{R} \cap X\right)$. 
Theorem 3.3. Let $F: \mathbb{C}^{n} \rightarrow \mathbb{C}^{n}$ has a nowhere zero Jacobian. Then the nonproperness set $S_{F}$ of $F$ can not have a trivial link at infinity.

Proof. Put $S_{F}:=X$. Since the mapping $F$ has finite fibers it is dominant, i.e., $\overline{F\left(\mathbb{C}^{n}\right)}=\mathbb{C}^{n}$. Moreover, if $X=\left\{x \in \mathbb{C}^{n}: h(x)=0\right\}$, then $h \circ F \neq$ const, and consequently $F^{-1}(X)$ is a hypersurface. Since $F$ is open, it implies that the set $F\left(\mathbb{C}^{n}\right) \cap X$ is dense in $X$. In particular, we can choose a point $a \in S_{R} \cap X$ in such a way that $a=F(b)$, where $b \in F^{-1}\left(S_{R}\right):=Y$, and additionally $a$ is a smooth point of $X$. Let $U_{a}$ be a small neighborhood of $a$ in $S_{R}$ such that on $U_{a}$ there exists a well defined branch $\psi$ of a mapping $F^{-1}$ such that $\psi(a)=b$. By Lemma 3.2 we can find a small loop $\gamma \subset U_{a}$, which generates $H_{1}\left(S_{R} \backslash S_{R} \cap X\right)=\mathbb{Z}$. Take a point $c \in \gamma$ and let $d=\psi(c)$. Note that the group $\pi_{1}\left(S_{R} \backslash S_{R} \cap X, c\right)$ is also generated by the loop $\gamma$.

By our first result the manifold $Y$ is connected and the mapping $F$ induces a covering $p: Y \backslash F^{-1}(X) \rightarrow S_{R} \backslash X$. Since $\gamma$ is the image of the loop $\gamma^{\prime}=\psi(\gamma)$ we have that the mapping $p_{*}: \pi_{1}\left(Y \backslash F^{-1}(X), d\right) \rightarrow \pi_{1}\left(S_{R} \backslash S_{R} \cap X, c\right)$ is an isomorphism. In particular the covering $p$ has topological degree

$$
d=\left[\pi_{1}\left(S_{R} \backslash S_{R} \cap X, c\right): p_{*} \pi_{1}\left(Y \backslash F^{-1}(X), d\right)\right]=1 .
$$

Thus the mapping $F$ induces trivial covering $Y \backslash F^{-1}(X) \rightarrow S_{R} \backslash X$. This implies that $F$ has topological degree one, consequently it is an isomorphism, a contradiction.

Acknowledgements The author is grateful to the anonymous referee for his helpful remarks.

Open Access This article is licensed under a Creative Commons Attribution 4.0 International License, which permits use, sharing, adaptation, distribution and reproduction in any medium or format, as long as you give appropriate credit to the original author(s) and the source, provide a link to the Creative Commons licence, and indicate if changes were made. The images or other third party material in this article are included in the article's Creative Commons licence, unless indicated otherwise in a credit line to the material. If material is not included in the article's Creative Commons licence and your intended use is not permitted by statutory regulation or exceeds the permitted use, you will need to obtain permission directly from the copyright holder. To view a copy of this licence, visit http://creativecommons.org/ licenses/by/4.0/.

\section{References}

[1] Benedetti, R., Risler, J.-J.: Real Algebraic and Semialgebraic Sets. Actualités Mathématiques, Paris (1990)

[2] Iitaka, S.: Algebraic Geometry. Springer, Berlin (1982)

[3] Jelonek, Z.: The set of points at which the polynomial mapping is not proper. Ann. Pol. Math. 58, 259-266 (1993)

[4] Jelonek, Z.: Testing sets for properness of polynomial mappings. Math. Ann. 315, 1-35 (1999)

[5] Jelonek, Z.: Geometry of real polynomial mappings. Math. Z. 239, 321-333 (2002) 
[6] Łojasiewicz, S.: Introduction to Complex Analytic Geometry. Springer, Berlin (1991)

[7] Nguen, T.: A condition for the properness of polynomial maps. Vietnam J. Math. 37, $113-125$ (2009)

[8] Rossi, H.: Vector fields on analytic spaces. Ann. Math. 78(3), 455-467 (1963)

[9] Sakkalis, T.: A note on proper polynomial maps. Commun. Algebra 33, 3359-3365 (2005)

[10] Zaidnberg, M.: On exotic algebraic structures on affine spaces. In: Noguchi, J. (ed.) Geometric Complex Analysis, pp. 691-714. World Sci. Publ. Co., Singapore (1996)

Publisher's Note Springer Nature remains neutral with regard to jurisdictional claims in published maps and institutional affiliations. 